\title{
ENERGY EFFICIENT LiNEAR CluSTER HANDLING PROTOCOL FOR WSN
}

\author{
Jaspinder Kaur ${ }^{1}$ and Varsha Sahni ${ }^{2}$ \\ ${ }^{1}$ Department of Computer Science Engineering, CTIEMT Shahpur Jalandhar, India
}

\begin{abstract}
Wireless sensor networks (WSNs) have attained expanding awareness from both the research association and authentic users. The efficient usage of energy source in a sensor node is very major criteria to sustain the life time of wireless sensor network. For obtaining durability of network lifetime, and reducing energy consumption, energy efficiency routing protocol play an important role. We are exhibit an innovative and energy efficient routing protocol in our research. Towards Energy Efficiency in Linear WSNs a linear cluster handling (LCH) technique is used with multiple static sinks in a linearly enhanced field of $1500 m * 350 m 2$. We are divided the whole into four equal sub-regions. We set multiple static sinks i.e. one at the centre and two at the both corners of the field for efficient data gathering. A reactive and Distance plus energy dependent clustering protocol Threshold Sensitive Energy efficient with Linear Cluster Handling [4] DE (TEEN-LCH) is implemented in the network field. Simulation shows improved results for our proposed protocol in term of throughput, packet delivery ratio and energy consumption.
\end{abstract}

\section{KEYWORDS}

WSN, Routing Protocols, nodes, energy

\section{INTRODUCTION}

A Wireless Sensor Networks (WSN) is a gathering of number of small sensor nodes that has power of construct wireless communication between each other, sensing and doing analysis and handle operations. Size and cost limitations on resource such as memory.

Applications of WSNs contained Enemy Tracking, Military Security Detection, Environment monitoring, traffic control etc. advanced routing techniques, like Minimum Transmission Energy (MTE) and Direct Communication (DC), as the fresh cluster based techniques these are not as energy-efficient, in the DC because every node send its sensed information directly to the sink . Nodes at a great from the BS die out more easily and lifetime of the network is reduced. MTE is better than DC because node communication with its nearest neighbor. Moreover, far nodes are disciplined by DC, whereas in MTE closer nodes battery potential drains out faster. To control all these limitations, which reduced the energy consumption the cluster based routing protocol; (LEACH) was proposed,

Efficiency and lifetime of WSNs depends upon the design [4] of the protocol. Packet Delivery Ratio, Energy Consumption, Throughput of the network is much improved in recent techniques [4]. In recent era, nearly all techniques chase the cluster based routing protocols. All nodes are deployed in the cluster and it receives data in their cluster, a $\mathrm{CH}$ is selected on the bases of energy and distance of the nodes in the cluster it receives data of all nodes and transfer it towards the

DOI : 10.5121/ijcsitce.2016.3201 
base station in the form of data packets, from station end user can access it easily. To reduce the exhaustion of energy, data aggregation is executed by the $\mathrm{CH}$. In this proposed scheme, extra data packets are sending to sink and existence of the network is enhanced.

In this paper, we establish a multi-sink protocol in a linearly enhanced field. We are deployed equal regions and equal number of nodes having similar quantity of energy in each region which formed homogenous network. While in our proposed protocol, multi-sink technique is used therefore, $\mathrm{CHs}$ in every region send their aggregated data to closest static sinks. Enhance the network lifetime and throughput of the network because of distribution of network into different regions and three static sinks on different places.

The next portion of the paper is order as follows: in section 2 described the related work. Section 3 described the motivation and points out the derivation of the recent works. The section 4 present about the proposed work. In section 5, simulations are discussed and different performance metrics are analyzed with their graphs. Then section 6 is about the conclusion of proposed technique.

\section{RELATED WORK}

This paper [1] carefully compares two different clustering protocols, namely LEACH and LEACH-C (centralized), using NS2 tool for many selected scenarios, and survey of simulation results against selected performance parameters with suspension and network lifetime being best among them. The paper will be finish by indicating the attention made from analyses of results about these protocols.

In this paper, [2] A General Self-Organized Tree-Based Energy-Balance routing protocol (GSTEB) is proposed by the authors, they are apply a procedure where, for each round, Sink allot a root node and forward this selection to all sensed nodes for makes a routing tree. eventually, each node chosen its parent by concede only itself and its neighbors' data, thus making GSTEB a dynamic protocol. Simulation results show that GSTEB shows a better results than other protocols in maintain energy consumption, thus increase the lifetime of WSN.

In this paper [3] the author for energy consumption concentrate on mainly driven over the survey of the hierarchical cluster-based available routing in Wireless Sensor Network . Low-Energy Adaptive Clustering Hierarchy $(\mathrm{LEACH})$ protocol is a good hierarchical protocols use the feasibility model to control the energy consumption of WSN. Simulation results, from distance to the Base Station, shows the energy consumption over time of three nodes.

In this paper, [4] authors propose a new scheme for chooses the sensors cluster-heads based on the quantity of energy remaining after each round [(4), (5)]. As the least rate of energy for the choose $\mathrm{d}$ leader is purposive in advance and accordingly limiting its performance and nonstop correlative task, to ensure reliable performance of the whole network used the new hierarchical routing protocol is based on an energy restrict value threshold avoiding the formation of a group leader.

In [5] authors deliberate that in wireless sensor network research the Routing protocol of is the main issue, the routing protocols can be branched into flat and hierarchical routing protocol according to network topology, . From the essential ideas, the article describes many classic hierarchical routing protocols in detail, the advantages and disadvantages and applications. 
In his paper, [6] the authors purpose Quadrature-LEACH (Q LEACH) for homogenous networks which increases network life-time, stability period, and throughput values.

In this paper [7], authors represent a scalable and energy efficient routing protocol, A New Linear Cluster Handling (LCH) [7]Technique Towards energy efficiency. In linear WSN in linearly enhanced field of $1000 \mathrm{~m} * 2 \mathrm{~m}$ with multiple static sinks. The whole linear field is separated into four equal sub-regions. For efficient data collection, place three static sinks i.e. two at the both corners and one at the centre of the field. There is types of routing protocols ,A proactive routing protocol Distributed Energy Efficient Clustering with Linear Cluster Handling (DEEC-LCH) is executed in the linear field. Moreover, a reactive protocol Threshold Sensitive Energy Efficient with same technique is also executed for the same scenario with three static sinks with different places. Simulation results shows better results for our proposed protocols as compared to basic DEEC and TEEN, in term of Throughput, network lifetime, and energy consumption.

\section{MOTIVATION}

In favor of enhance the lifetime of network, there are used mostly two possible approaches. The first approach is to reduce the limit of data, but this would minimize the throughput. The second option is to extend the energy of sensor nodes that becomes the device pricier. The better related routing protocol as compared to DC and MTE, In the previous scheme [4]in a linearly enhanced field of $1000 \mathrm{~m} * 2 \mathrm{~m}^{2}$ a linear cluster handling (LCH) scheme regarding Energy Efficiency in Linear WSNs with no. of static sinks on different places. They are divided the whole into four equal sub-regions. For efficient data gathering, they place three static sinks i.e. one at the centre and two at the both corners of the field. In our proposed protocol, we are chosen cluster head on the basis of energy and distance of nodes in each sub-region and advertisement is done by sink only in first round instead of in every round which is enhanced the network efficiency as compared to previous technique.

\section{THE PROPOSED PROTOCOL}

In this segment, we discus and implementation about our proposed protocol DE (TEEN-LCH) in which the $\mathrm{CH}$ is selected depend of DE ratio of the nodes in the network field. Description of DE (TEEN-LCH) is given in the following subsections.

\begin{tabular}{|c|c|}
\hline $\begin{array}{c}\text { No. of Item description } \\
\text { specification }\end{array}$ & No. of Item description specification \\
\hline Simulation Area & $1500 \mathrm{~m} * 350 \mathrm{~m}^{2}$ \\
\hline No. of nodes & 47 \\
\hline Channel type & Channel/Wireless Channel \\
\hline Simulation time & $35.0 \mathrm{sec}$ \\
\hline Antenna model & Antenna/Omni Antenna \\
\hline Link Layer Type & LL \\
\hline Energy Model & Energy Model \\
\hline MAC type & Mac/802_11 \\
\hline Interface queue type & Queue/ Drop Tail/Pri Queue \\
\hline $\begin{array}{c}\text { radio-propagation } \\
\text { model }\end{array}$ & Propagation/Two Ray Ground \\
\hline network interface type & Phy/Wireless Phy \\
\hline
\end{tabular}




\subsection{Region Formation}

In order to perform proper transmitting of data packets using multiple sinks, proposed protocol is linearly enhanced in the field of $1500 \mathrm{~m} \times 350 \mathrm{~m}^{2}$. We split whole network area into equal sized sub-regions. In each sub-region, an independent cluster is created and the cluster head is formed on the basis of ratio of energy and distance which eventually decrease the transmission distance as well as energy consumption.

\subsection{Deployment of Nodes and Sinks Position}

The major task behind sub-region formation is to employ nodes in the field in such a way that maximum field can be bounded by the nodes. Equal numbers of nodes are employed in each subregion. There are three base station are placed in the network, two at the both corners of the field and one at the middle of the field. In this way, $\mathrm{CH}$ receives sensed data of the nodes and sends it to its nearby sink in the field.

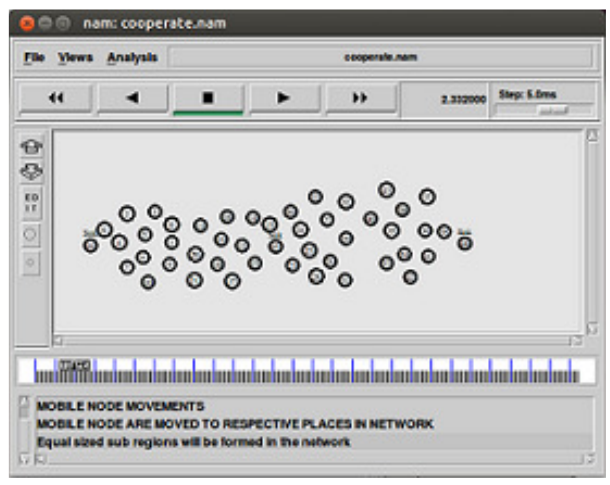

Fig 1: Region formation and Sink placement. 


\subsection{Protocol operation}

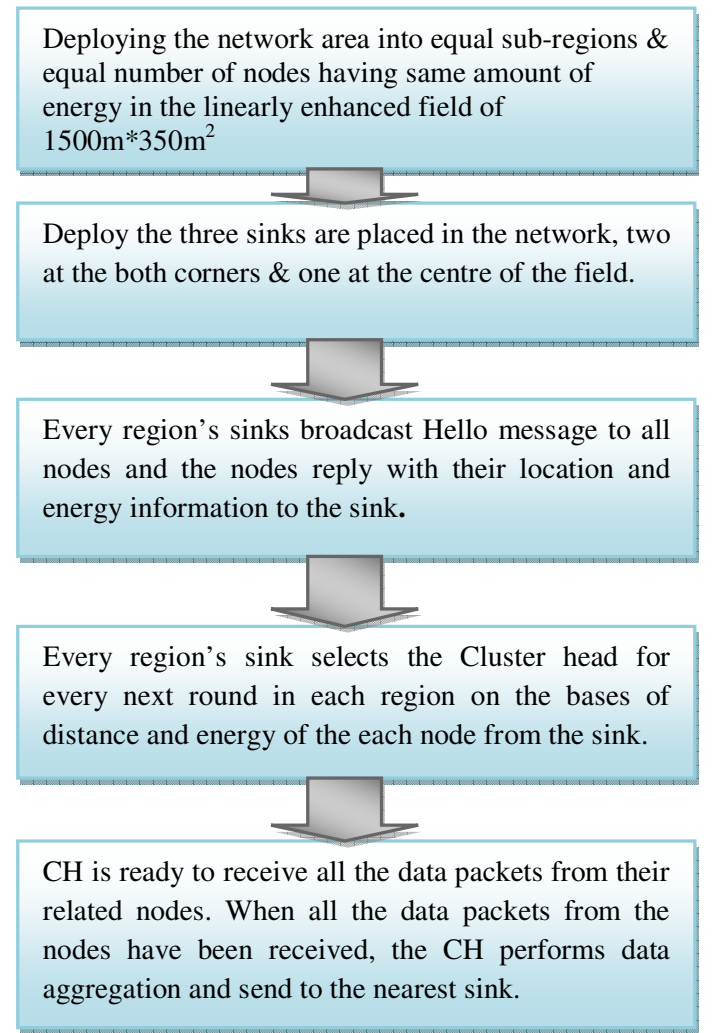

The protocol working is divided into separate phases such as;

- Advertisement Phase

- $\quad$ Cluster setup phase

- Data transmission phase

\subsection{Advertisement Phase}

The proposed protocol earns credit by implementation multi-sinks and cluster formation in each region, which result in extension of throughput and lifetime of the network. Every region's sinks broadcast Hello message to all nodes and the nodes reply with their location and energy information to the sink. The sink selects the cluster head for every next round by using this information.

\subsection{Cluster phase setup}

Initially, when clusters are formed in a region, each node sends their location and energy information in the reply of sink's hello broadcast message, and then. The sink select the node as a cluster head whose energy is more and the distance is less from the sink. 


\subsection{Data Transmission}

Once sub-regions are formed and clusters are selected, then data transmission is started. $\mathrm{CH}$ is ready to receive all the data packets from their related nodes. When all the data packets from the nodes have been received, the $\mathrm{CH}$ performs data aggregation and send to the sink. As the Base Station is nearby every sub-region, so it requires low transmission energy. Same procedure is executed in every sub-region.

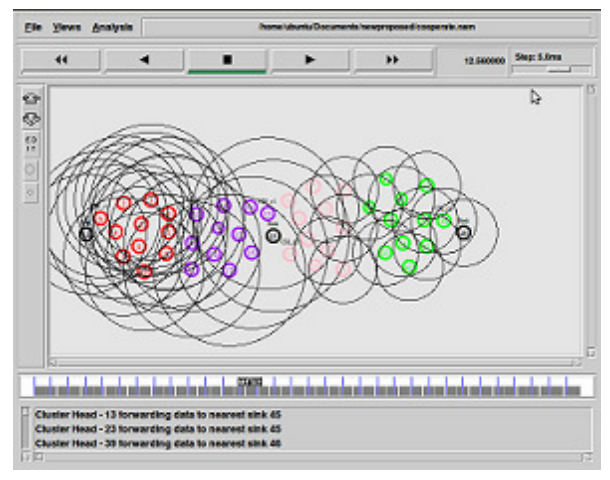

Fig 2: Cluster Setup and data transmission.

\section{SIMULATION RESULTS}

Performance of proposed protocol DE (TEEN-LCH) is representing on the basis of different parameters. Whole region of $1500 \times 350 \mathrm{~m}^{2}$ is divided into four sub-regions in which equal number of nodes is randomly deployed. Three sinks are placed in the network at different locations and Cluster Head of every sub-region sends data packets to its closest sink respectively.

\subsection{Throughput}

Throughput is the average of data packets received at the destination (i.e. at base station). Throughput=received data $* 8$ / data transmission period

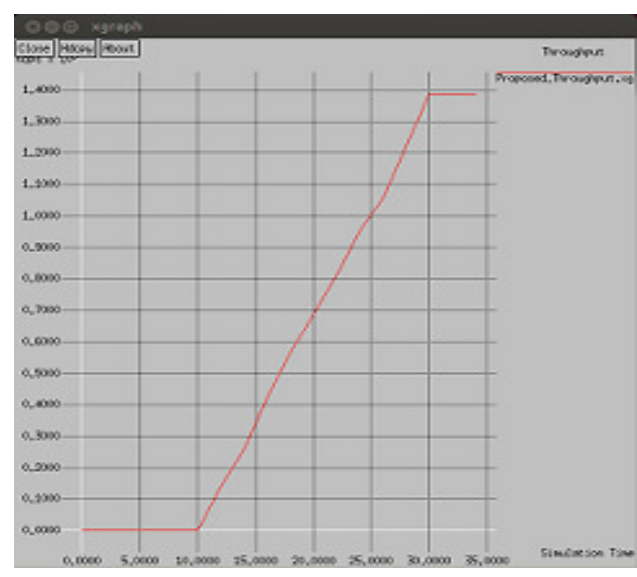

Fig 3: Throughput. 
TABLE I.

\begin{tabular}{|c|c|}
\hline \multicolumn{1}{|l|}{ TIME } & DE(TEEN-LCH)(THROUGHPUT) SEC \\
\hline $0-10$ & 0 \\
\hline 12 & 108.544 \\
\hline 14 & 260.096 \\
\hline 16 & 425.984 \\
\hline 18 & 581.632 \\
\hline 20 & 706.56 \\
\hline 22 & 827.392 \\
\hline 24 & 974.848 \\
\hline 26 & 1073.15 \\
\hline 28 & 1234.94 \\
\hline 30 & 1398.78 \\
\hline 32 & 1400.83 \\
\hline 34 & 1400.83 \\
\hline
\end{tabular}

\subsection{Energy Consumption}

The energy consumption is the performance matrices that summation of used energy by all the nodes in the network field, where the energy utilization of a node is the aggregation of the energy used for transmission, along with sending, receiving, and idling. Assuming each transmission spend an energy unit, the whole energy exhaustion is equal to the total no. of data packets broadcast in the network field. Energy $\{\exp \$$ initial energy $(\$ i)-\$$ final energy $(\$ f)\}$

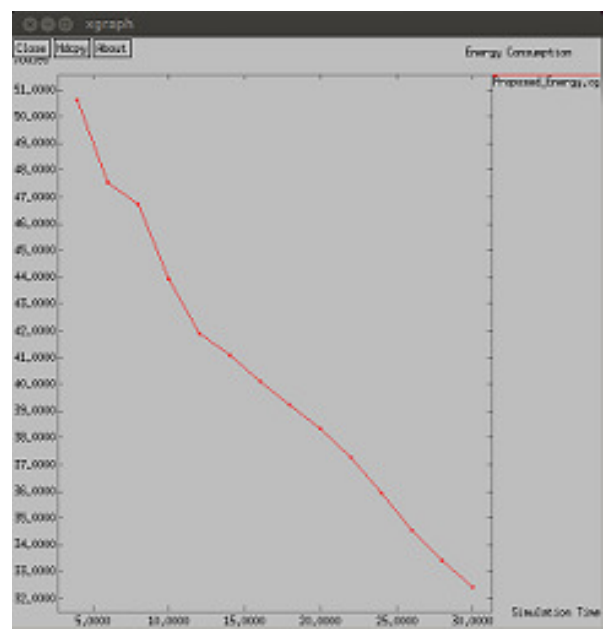

Fig 4: Energy Consumption 
TABLE II.

\begin{tabular}{|c|c|}
\hline TIME & DE(TEEN-LCH) (ENERGY CONSUMPTION) JOULES \\
\hline 4 & 50.6342 \\
\hline 6 & 47.5383 \\
\hline 8 & 46.7354 \\
\hline 10 & 43.9388 \\
\hline 12 & 41.9243 \\
\hline 14 & 40.984 \\
\hline 16 & 40.1595 \\
\hline 18 & 39.2621 \\
\hline 20 & 38.2221 \\
\hline 22 & 37.0416 \\
\hline 24 & 35.3813 \\
\hline 26 & 34.4253 \\
\hline 28 & 33.2566 \\
\hline 30 & 31.5012 \\
\hline & \\
\hline &
\end{tabular}

\subsection{Packet Delivery ratio}

It is represent as the ratio of no. of data packets received to the no. of data packets sent in the network to the sink. The larger value of the data packet delivery ratio means improved performance of protocol.

PDR = received_packets / generated_packets

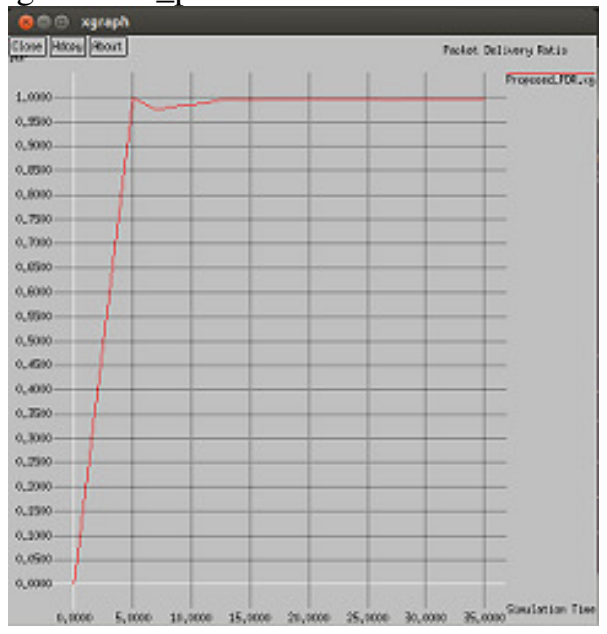

Fig 5: Packet Delivery ratio 
TABLE III.

\begin{tabular}{|c|c|}
\hline \multicolumn{1}{|l|}{ TIME } & DE(TEEN-LCH )(PDR) \% \\
\hline 0 & 0 \\
\hline 5 & 1 \\
\hline 7 & 0.974359 \\
\hline 9 & 0.982456 \\
\hline 11 & 0.986486 \\
\hline 13 & 0.99639 \\
\hline 15 & 0.997807 \\
\hline 17 & 0.998624 \\
\hline 19 & 0.998848 \\
\hline 21 & 0.999055 \\
\hline 23 & 0.999108 \\
\hline 25 & 0.999242 \\
\hline 27 & 0.998535 \\
\hline 29 & 0.99873 \\
\hline 31 & 0.998854 \\
\hline 33 & 0.998854 \\
\hline 35 & 0.998854 \\
\hline
\end{tabular}

\section{CONCLUSION}

We proposed DE (TEEN-LCH) an energy-aware adaptive multi-sink routing protocol used in linearly enhanced field. In each region equal numbers of nodes are randomly deployed. Three sink are placed on the three different places in the network these sink receive data packets from their nearest nodes and CHs. We proposed a new DE (TEEN-LCH) an energy-aware adaptive multi-sink routing protocol used in linearly enhanced field. The $\mathrm{CH}$ is chooses by the each region's Base Station in every region for each round with the help of advertisement phase in which the sink is send hello message to the nodes and nodes reply with their location and energy information and then sink select the cluster head based on DE ratio. In the same way, results present that the proposed strategy increases the packet delivery ratio and improves the throughput. In future, we are interested to implement mobile sinks with chain based routing.

\section{AKNOWLEDGMENT}

The authors say to thank the faculty members for their continued support and feedback from the computer science department at CTIEMT, Jalandhar.

\section{REFERENCES}

[1] Geetha. V., Pranesh.V. Kallapur, Sushma Tellajeera," Clustering in Wireless Sensor Networks: Performance Comparison of LEACH \& LEACH-C Protocols Using NS2” ELSEVIER Procedia Technology 4 ( 2012 ) 163 170

[2] Zhao Han, Jie Wu, Member, IEEE, Jie Zhang, Liefeng Liu, and Kaiyun Tian, "A General Self-Organized TreeBasedEnergy-Balance Routing Protocol” IEEE TRANSACTIONS ON NUCLEAR SCIENCE, VOL. 61, NO. 2, APRIL 2014

[3] Shanthi Dr. E.RamaDevi "A Cluster Based Routing Protocol in Wireless Sensor Network for Energy Consumption” Int. J. Advanced Networking and Applications Volume: 05, Issue: 04, Pages:2015-2020 (2014) ISSN : 0975-0290 
[4] Salim EL KHEDIRIa,b,c, Nejah NASRIa, Anne WEIc, Abdennaceur KACHOURId," A New Approach for Clustering in Wireless Sensors Networks Based on LEACH” ELSEVIER Procedia Computer Science 32 ( 2014 ) $1180-1185$

[5] DaWei Xu, Jing Gao,a "Comparison Study to Hierarchical Routing Protocols in Wireless Sensor Networks" ELSEVIER Procedia Environmental Sciences 10 ( 2011 ) 595 - 600

[6] B. Manzoor, N. Javaid, O. Rehman, M. Akbar, Q. Nadeem, A. Iqbal, M. Ishfaq" Q-LEACH: A New Routing Protocol for WSNs " Procedia Computer Science 19 ( 2013 ) 926 - 931

[7] M. Sajid, K. Khan, U. Qasim, Z.A. Khaan,S. Tariq, N. Javaid,"A New Linear Cluster Hnadling(LCH)Technique Toward's Energy efficiency in Linear WSNs" 2015 IEEE 29 International Conference on Advanced Information Networking and Applications.

[8] Jaspinder Kaur, Varsha Sahni "Survey on Hierarchical Cluster Routing Protocols of WSN" International Journal of Computer Applications (0975 - 8887) Volume 130 - No.17, November2015

[9] F. Xiangning, S. Yulin. "Improvement on LEACH Protocol of Wireless Sensor Network", 2007, International Conference on Sensor Technologies and Applications pp 260-264, ido:10.1109/SENSORCOMM.2007.60

[10] Kemal Akkaya , Mohamed Younis "A survey on routing protocols for wireless sensor networks" ELSEVIER,

[11] Jaspinder Kaur, Taranvir Kaur, Kanchan Kaushal "Survey on WSN Routing Protocols" International Journal of Computer Applications (0975 - 8887) Volume 109 - No. 10, January 2015

[12] Varsha Sahni ,Jaspreet Kaur, Sonia Sharma "Security Issues and Solutions in Wireless Sensor Networks" International Journal of Computer Science and Information Technologies, Vol. 3 (2), 2012,

[13] Nikolaos A. Pantazis, Stefanos A. Nikolidakis and Dimitrios D.Vergados, Senior Member, IEEE" EnergyEfficient Routing Protocols in Wireless Sensor Networks: A Survey” IEEE communications survey \& tutorials, vol. 15, no. 2, second quarter 2013

[14] Harjeet Kaur 1, Manju Bala 2 ,Varsha Sahni 3 "Study of Black hole Attack Using Different Routing Protocols in MANET" International Journal of Advanced Research in Electrical, Electronics and Instrumentation Engineering, Vol. 2, Issue 7, July 2013

[15] Sanjeev Kumar Gupta, Neeraj Jain, Poonam Sinha "Clustering Protocols in Wireless Sensor Networks: A Survey" International Journal of Applied Information Systems (IJAIS) - ISSN : 2249-0868 Foundation of Computer Science FCS, New York, USA Volume 5- No.2, January 2013

[16] S. Misra et al. (eds.), Guide to Wireless Sensor Networks, Computer Communications and Networks, DOI: 10.1007/978-1-84882-218-4 4, Springer-Verlag cAst2weszvxvx London Limited 2009.

[17] Manju Bala , Lalit Awasthi "On Proficiency of HEED Protocol with Heterogeneity for Wireless Sensor Networks with BS and Nodes Mobility" International Journal of Applied Information Systems (IJAIS) - ISSN : 2249-0868 Foundation of Computer Science FCS, New York, USA Volume 2- No.7, May 2012

[18] Ming Zhang, Yanhong Lu, Chenglong Gong, " Energy-Efficient Routing Protocol based on Clustering and Least Spanning Tree in Wireless Sensor Networks", International Conference on Computer Science and Software Engineering,IEEE, 2008.

[19] Jennifer Yick , Biswanath Mukherjee, Dipak Ghosal“ On the Wireless sensor network survey” ELSEVIER, Computer Networks 52 (2008) 2292-2330 